\title{
Cálculo vesical e nefrolitíase bilateral em cão: relato de caso
}

Catarina Rafaela Alves da Silva ${ }^{*}$, Francisco Lima Silva ${ }^{2}$, Elisângela de Sá ${ }^{3}$, Marllos Henrique Vieira Nunes ${ }^{3}$, Hannah Martins Oliveira Gonçalves da Silva ${ }^{4}$, Paulo Victor Garrêto Rodrigues dos Santos ${ }^{5}$, Huanna Waleska Soares Rodrigues ${ }^{3}$, Joice Rayane de Alencar Oliveira ${ }^{4}$, Lianna Martins Oliveira Gonçalves da Silva ${ }^{3}$

\author{
${ }^{1}$ Professora do Departamento de Clínica e Cirurgia Veterinária da Universidade Federal do Piauí UFPI. \\ ${ }^{2}$ Professor do Departamento de Clínica e Cirurgia Veterinária da UFPI. \\ ${ }^{3}$ Graduando (a) Medicina Veterinária da UFPI. \\ ${ }^{4}$ Médica veterinária residente de clínica e cirurgia do Hospital Veterinário Universitário/UFPI \\ ${ }^{5}$ Médico Veterinário Autônomo. \\ *Email correspondência: catarinarafaela@hotmail.com
}

\begin{abstract}
RESUMO. A urolitíase é uma enfermidade que acomete cães e gatos, em resultado de processos multifatoriais para seu desenvolvimento ocasionando obstrução do fluxo urinário, presença de infecção no trato urinário e até mesmo evoluir para o óbito do animal se caso não tratada. A formação de cristais e cálculos tem como causas a diminuição na frequência urinária associada à supersaturação e mudança de $\mathrm{pH}$ da urina, podendo estar envolvido a fatores dietéticos ou até no manejo. A nutrição pode estar relacionada à formação, prevenção e tratamento das urolitíases.
\end{abstract}

Palavras-chave: cristais, nefrotomia, canino, rim, urólito.

\section{Bladder calculus and bilateral nephrolithiasis in dog: a case report}

\begin{abstract}
Urolithiasis is a disease that affects dogs and cats, as a result of multifactorial processes for their development causing urinary outflow obstruction, presence of urinary tract infection and even evolve to death if the animal if left untreated. The formation of crystals and has as calculation causes a decrease in urinary frequency associated with a change in $\mathrm{pH}$ and supersaturation of urine, which may be the dietary factors involved in the management or even. Nutrition may be related to training, prevention and treatment of urolithiasis.
\end{abstract}

Keywords: crystals, nefrotomy, canine, kidney, urolith

\section{Introdução}

Urolitíase é a formação de precipitados em forma sólida em qualquer ponto das vias urinárias em decorrência da precipitação de minerais ou substâncias orgânicas no trato urinário (Assis et al., 2009). A maioria dos urólitos nos cães é encontrada na bexiga ou uretra, sendo que apenas $5 \%$ é encontrado nos ureteres ou rins (Grauer, 2011). A formação dos urólitos está relacionada a fatores dietéticos e não dietéticos. Entre os não dietéticos estão a raça, idade, infecção do trato urinário, sexo, cães de pequeno porte. $\mathrm{O}$ fator dietético está relacionado à composição da dieta, uma vez que esta pode afetar a densidade específica volume e pH urinário (Carciofi et al., 2007). Os sinais clínicos mais observados são polaciúria, disúria, estrangúria, hematúria, incontinência urinária, uremia (Grauer, 2011). A urolitíase canina é diagnosticada baseando-se na anamnese, exame físico e exames complementares (Morschbacher et al., 2008). O tratamento da afecção pode ser clínico, através da dissolução e/ou interrupção do crescimento subsequente de urólitos, ou cirúrgico, através da remoção dos mesmos. A remoção cirúrgica nos casos de nefrólitos é indicada nos casos não tratáveis apenas com a dissolução dietética, possíveis obstrução do trato urinário, aumento progressivo do urólitos e rim contralateral afuncional (Filgueira et al., 2010). A profilaxia baseia-se no conhecimento da composição mineral dos mesmos, a fim de se evitar recorrências (Fossum et al., 2008). 


\section{Descrição do caso}

Um cão, SRD, sete anos foi atendido em uma clínica particular, apresentando quadro clínico de vômitos, hematúria, disúria e inapetência há aproximadamente 6 dias. Alimentava-se de ração comercial duas vezes ao dia. Ao exame físico, observou-se sensibilidade dolorosa à palpação da região abdominal e sublombar. Foram solicitados exames complementares, tais como: hemograma (Anemia normocítica normo-crômica, trombocitopenia, leucopenia com linfopenia e monocitopenia absolutas, neutrofilia relativa e aneosinofilia). No exame bioquímico (uréia $=$ $309 \mathrm{mg} / \mathrm{dl}$, creatinina $=5,16 \mathrm{mg} / \mathrm{dl}, \mathrm{ALT}=26 \mathrm{U} / \mathrm{l}$, $\mathrm{FA}=49 \mathrm{U} / \mathrm{l}, \quad \mathrm{PT}=4,2 \mathrm{~g} / \mathrm{dl})$. No exame ultrassonográfico foi constatado aumento nos rins bilateral e consequente estrutura hiperecoica formadora de sombra acústica e também visível na bexiga. No exame radiográfico simples foi visualizada estrutura radiopaca nos rins direito e esquerdo e bexiga. Optou-se então pelo tratamento cirúrgico. Como MPA foi utilizado tramadol $2 \mathrm{mg} / \mathrm{kg} / \mathrm{IV}$ e diazepam $0,5 \mathrm{mg} / \mathrm{kg} / \mathrm{IV}$. A indução consistiu em $5 \mathrm{mg} / \mathrm{kg} / \mathrm{IV}$ propofol, dose efeito e manutenção por Isofluorano via inalatória em oxigênio a $100 \%$.

A cirurgia realizada foi nefrotomia por meio de celiotomia mediana ventral, sendo que ambos nefrólitos localizavam-se na pelve renal e cistotomia para a retirada do urólito presente na vesícula urinária.

O pós-operatório constou de fluidoterapia e uso de antibiótico (Amoxicilina com Clavulanato de potássio $20 \mathrm{mg} / \mathrm{kg} / \mathrm{BID}$ ), terapia analgésica e antiinflamatória (Dipirona $25 \mathrm{mg} / \mathrm{kg} / \mathrm{TID}$, Meloxicam 0,1 mg/kg/SID). Como o animal apresentou hematúria, 5 dias após cirurgia desenvolveu um quadro de uremia com a permanência da ureia e creatinina altas, sendo indicada a eutanásia.

\section{Discussão}

A urolitíase não é uma enfermidade isolada, com único agente etiológico, mas sim como consequência de múltiplas anormalidades, incluindo fatores raciais, congênitos, ou patofisiológicos adquiridos que, em combinação, incrementam o risco de precipitação dos metabólitos excretados pela urina, podendo ser encontrados em qualquer porção do trato urinário (Neta; Munhoz, 2008). Representam cerca de $18 \%$ das afecções urinárias em cães (Monferdini et al., 2009). Os sólidos que se formam no trato urinário são anormais, pois o sistema urinário é designado para eliminar os resíduos metabólicos na forma líquida. Das alterações mais comuns do trato urinário tem se a falha na eliminação dos metabólitos corporais, que se acumulam na forma de inúmeros precipitados. Dentre esses precipitados, são de considerável importância os cristais (Neta \& Munhoz, 2008). A formação de cristais e urólitos têm como principais causas a diminuição na frequência urinária associada à supersaturação, mudança de $\mathrm{pH}$ urinário, infecção de trato urinário, alta concentração de cristaloides na urina e fatores dietéticos que isolados ou em conjunto predispõe à sua formação (Fossum et al., 2008; Sturion et al., 2011). Os animais podem ter urólitos em mais de um local, sendo que na maioria dos cães são encontrados na bexiga (urocistólitos) ou na uretra (uretrólitos); somente de 5\% a 10\% se localizam nos rins (nefrólitos) ou nos ureteres (ureterólitos) (Neta \& Munhoz, 2008). Neste caso relatado optou-se na realização de nefrotomia devido à localização do nefrólito, bilateral e ainda presença de urólito na bexiga. Segundo Dall' Asta et al. (2011) a nefrotomia é indicada nos casos de nefrólitos com constituição de oxalato de cálcio e com dimensões significativas.

\section{Conclusão}

A urolitíase não é uma afecção primária, mas uma consequência secundária a várias desordens sistêmicas. A indicação cirúrgica é somente em casos em que a dieta não consegue atingir o resultado esperado e em casos graves, como nefrólito bilateral associado com urólito na vesícula urinária. Embora tenha se empregado a cirurgia para o tratamento neste relato, as taxas bioquímica séricas de creatinina e ureia e uma grande lesão os órgãos alvo, impossibilitaram o animal de responder ao tratamento pós-cirúrgico, sendo indicada a eutanásia.

\section{Referências bibliográficas}

Assis, A. C. O; Silva, T. R; Aguiar, G. M. N; Melo D. B; Almeida, F. C; Medeiros, J. M \& Nóbrega Neto, P. I. Urolitíase Obstrutiva em Bovinos no semiárido Paraibano. In: VIII Congresso Brasileiro de Buiatria. Anais. Belo Horizonte, 2009.

Carciofi, A. Como a dieta influencia o $\mathrm{pH}$ urinário e a formação de cálculos em cães e gatos? In: Anais do Simpósio sobre nutrição de animais de estimação. Campinas, 2007. 
Dall'Asta, L. B.; Reolon, M.; Noronha, F. \& Martins, D. B. Urolitíase em um canino relato de caso. XVI Seminário Interinstitucional de Ensino, Pesquisa e Extensão. Out/2011

Grauer, G. F. Urolitíase canina. In: Nelson, R.W \& Couto, C. G. Manual de Medicina Interna de Pequenos Animais. 2ed. Rio de Janeiro: Elsevier, p.468,2011.

Filgueira, G. F. F.; Carneiro, R. S.; Nunes, G. D. L.; Fernandes, T. H. T.; Araújo, A, L.; Leite, A. R. A.; Pedrosa, D. \&Neto, P. I. N. Urolitíase vesical em gata - diagnóstico e tratamento. X Jornada de Ensino, Pesquisa e Extensão, UFRPE, 2010.

Fossum, T. W.; Duprey, L. P.; O'Connor, D. Cirurgia de pequenos animais. $3^{\mathrm{a}}$ Ed. Rio de Janeiro: Elsevier, 2008.

Monferdini, R. P. \& Oliveira, J. Manejo nutricional para cães e gatos com urolitíase Revisão bibliográfica. Acta Veterinária Brasileira. n. 1. v. 3. p. $1-4,2009$.

Morschbacher, P. D.; Correa, R. K. R.; Barbosa, F. C.; Rigon, G. M. \& Stedile, R. Remoção de urolitíase por nefrotomia em um cão- relato de caso.In: Congresso Brasileiro de Medicina Veterinária. Anais. Gramado, 2008.

Neta, E. S. M \& Munhoz, A .D. Urolitíase em cães e gatos: Uma revisão. MEDVEP Revista Científica Veterinária de Pequenos Animais de Estimação, n.6, vol.17, p.24-34, 2008.

Sturion, D. J.; Sturion, M. A. T.; Sturin, T. T.; Saliba, R.; Martins, E. L.; Silva, S. J. \& Costa, M. R. Urolitíase em cães e gatos - revisão de literatura. Fio. Edu. Bras., 2011

Recebido em Agosto 22, 2014.

Aceito em Novembro 11, 2014

License information: This is an open-access article distributed under the terms of the Creative Commons Attribution License, which permits unrestricted use, distribution, and reproduction in any medium, provided the original work is properly cited 